PROCEEDINGS OF THE

AMERICAN MATHEMATICAL SOCIETY

Volume 138, Number 4, April 2010, Pages 1361-1370

S 0002-9939(09)10154-5

Article electronically published on November 2, 2009

\title{
EXACT CONTROLLABILITY OF GALERKIN'S APPROXIMATIONS OF MICROPOLAR FLUIDS
}

\author{
F. D. ARARUNA, F. W. CHAVES-SILVA, AND M. A. ROJAS-MEDAR
}

(Communicated by Walter Craig)

\begin{abstract}
We consider the nonlinear model describing micropolar fluid in a bounded smooth region of $\mathbb{R}^{N}(N=2,3)$ with distributed controls supported in small subset of this domain. Under suitable assumptions on the Galerkin basis, we introduce Galerkin's approximations for the controllable micropolar fluid system. By using the Hilbert Uniqueness Method in combination with a fixed point argument, we prove the exact controllability result for this finitedimensional system.
\end{abstract}

\section{INTRODUCTION}

Let $\Omega \subset \mathbb{R}^{3}$ be a bounded open set whose boundary $\Gamma$ is sufficiently regular. Let $\mathcal{O} \subset \Omega$ be a (small) nonempty open subset. For $T>0$, we consider the cylindrical domain $Q=\Omega \times(0, T)$ of $\mathbb{R}^{4}$ with lateral boundary $\Sigma=\Gamma \times(0, T)$. By $\boldsymbol{\nu}=\boldsymbol{\nu}(x)$ we denote the outward unit normal vector to $\Omega$ at the point $x \in \Gamma$.

We adopt the notations $\mathbf{y}, \mathbf{w}$ and $p$ for the velocity field, the angular velocity of rotation of the fluid particles, and the pressure distribution, respectively. Spaces of $\mathbb{R}^{3}$-valued functions, as well as their elements, are represented by bold-face letters. The governing equations are the following:

$$
\begin{cases}\mathbf{y}_{t}-\left(\mu+\mu_{r}\right) \Delta \mathbf{y}+(\mathbf{y} \cdot \nabla) \mathbf{y}+\nabla p=2 \mu_{r} \nabla \times \mathbf{w}+\mathbf{u} 1_{\mathcal{O}} & \text { in } Q \\ \mathbf{w}_{t}-\widehat{c} \Delta \mathbf{w}+(\mathbf{y} \cdot \nabla) \mathbf{w}-\bar{c} \nabla(\nabla \cdot \mathbf{w})=2 \mu_{r} \nabla \times \mathbf{y}+\mathbf{v} 1_{\mathcal{O}} & \text { in } Q \\ \nabla \cdot \mathbf{y}=0 & \text { in } Q \\ \mathbf{y}=\mathbf{w}=0 & \text { on } \Sigma, \\ \mathbf{y}(0)=\mathbf{y}^{0}, \quad \mathbf{w}(0)=\mathbf{w}^{0} & \text { in } \Omega,\end{cases}
$$

where $\widehat{c}=c_{a}+c_{d}$ and $\bar{c}=c_{0}+c_{d}-c_{a}$.

In (1.1), $\mathcal{O}$ is the control domain (which is supposed to be as small as necessary), $\mathbf{u}$ and $\mathbf{v}$ are the control functions which act over the system, $1_{\mathcal{O}}$ denotes the characteristic function of $\mathcal{O}$, and the positive constants $\mu, \mu_{r}, c_{0}, c_{a}$ and $c_{d}$ characterize the physical properties of the fluid: $\mu$ is the usual Newtonian viscosity and $\mu_{r}, c_{0}$,

Received by the editors March 30, 2009, and, in revised form, July 1, 2009, July 14, 2009, and July 29, 2009.

2000 Mathematics Subject Classification. Primary 35Q35, 93B05; Secondary 65M60.

Key words and phrases. Micropolar fluids, controllability, Galerkin approximations.

The first author was supported by CNPq-Brazil and FAPESQ-PB-Brazil.

The second author was supported by CAPES-Brazil.

The third author was partially supported by Fondecyt-Chile, Grant 1080628 and MTM200607932, Spain. 
$c_{a}, c_{d}$ are additional viscosities related to the lack of symmetry of the stress tensor and, consequently, to the fact that the internal rotation $\mathbf{w}$ does not vanish. These constants satisfy the physically relevant inequality $c_{0}+c_{d}>c_{a}$.

For the model derivation and related physical discussion, see Condiff and Dalher [5], Eringen 9], 10, Lukaszewicz [24] and Petrosyan [26]. We observe that (1.1) includes as a particular case the classical Navier-Stokes equations, which have been widely studied (see, for instance, Ladyzhenskaya 19 or Temam 30 and the references therein). In this case $\mu_{r}=0$ and equations $(1.1)_{1}$ and $(1.1)_{2}$ are decoupled. Several experiments show that solutions of the micropolar fluid model describe behavior of numerous real fluids better than corresponding solutions of the Navier-Stokes equations. In particular, this nonlinear coupled system also can be used to simulate the behavior of liquid crystals, polymeric fluids and blood in thin vessels (see [1, 4], 8, 17, [27, 29] and the references therein).

A good reference for the mathematical aspects of (1.1) is [24]; it contains important existence and uniqueness results. In 28] the existence of a strong solution is proved using the Galerkin method, and in 25] the regularity results for weak solutions is proved.

Due to the dissipative and nonreversible properties of the system, it is clear that one cannot expect the exact controllability for the micropolar fluid model with arbitrary target functions (in the same way as in Navier-Stokes equations). On the other hand, approximate controllability is an open question for this one. However, the notion of approximate controllability does not appear to be really meaningful. Indeed, even if we could reach an arbitrary neighborhood of a given target $\left\{\mathbf{y}^{T}, \mathbf{w}^{T}\right\}$ at time $T$ by the action of a control, the question of what to do after time $T$ to stay in the same neighborhood would remain open.

In the context of the Navier-Stokes equations, there are results of the local exact controllability to uncontrolled trajectories obtained in Fursikov-Imanuvilov [16, Imanuvilov [18, and Fernández-Cara et al. [12. The global approximate controllability of the 2-D Navier-Stokes equations with slip boundary conditions was obtained by Coron [6]. Combining results on global approximate controllabillity and local controllability, the global exact controllability for the Navier-Stokes system on a 2-D manifold was analyzed in Coron-Fursikov [7. In [21] and 22, Lions and Zuazua introduced the finite-dimensional Galerkin's approximations for the NavierStokes system, and they proved that these Galerkin's approximations are exactly controllable. Optimal control problems associated with the Navier-Stokes equations also have a wide and important range in applications. This issue has been studied, for instance, by Fursikov in [13] and by Gunzburger and Hou in [14] and [15].

Concerning control results for the micropolar fluids, Fernández-Cara and Guerrero in [11] studied the local exact controllability to the trajectories for system (1.1). Notice that this case involves a difficulty. The main reason is that $\mathbf{w}$ is a nonscalar variable and the equations satisfied by its components $w_{i}$ are coupled through the second-order terms $\partial_{i}(\nabla \cdot \mathbf{w})$. In the present work, we study the exact controllability for the micropolar fluids approached by Galerkin's approximations, and we reach basically the same level of knowledge as in the case of the classical Navier-Stokes equations. The results given in our work can be applied to different models of fluid mechanics. Some of these models, such as generalized Boussinesq systems (see [2]) and equations describing liquid crystals (see [17]) are intensively studied. 
This paper is organized as follows: In Section 2, we state the basic notations. In Section 3, we introduce Galerkin's approximations for (1.1) and, for this finitedimensional system, we establish the exact controllability result. The proof is based on the Hilbert Uniqueness Method introduced by Lions (see, for instance, 20] ) to study the exact controllability of linear systems and a fixed point technique.

\section{Preliminaries}

The following vector spaces, usual in the context of incompressible fluids, will be used throughout the paper:

$$
\mathbf{V}=\left\{\boldsymbol{\varphi} \in \mathbf{H}_{0}^{1}(\Omega) ; \nabla \cdot \varphi=0 \text { in } \Omega\right\}
$$

and

$$
\mathbf{H}=\left\{\boldsymbol{\varphi} \in \mathbf{L}^{2}(\Omega) ; \nabla \cdot \boldsymbol{\varphi}=0 \text { in } \Omega, \boldsymbol{\varphi} \cdot \boldsymbol{\nu}=0 \text { on } \Gamma\right\} .
$$

Since the constants $\mu, \mu_{r}, c_{a}, c_{d}$ and $c_{0}$ are not relevant in the arguments and results, we set in (1.1) all coefficients equal to one.

Here and in what follows $(\cdot, \cdot)$ and $\|\cdot\|$ stand for the scalar product and the norm in $\mathbf{L}^{2}(\Omega)$, respectively, and we denote

$$
a(\mathbf{u}, \mathbf{v})=\sum_{i, j=1}^{3} \int_{\Omega} \frac{\partial u_{i}}{\partial x_{j}} \frac{\partial v_{i}}{\partial x_{j}} d x .
$$

We will use $C$ to denote a generic positive constant which may vary from line to line (unless otherwise stated).

Multiplying (1.1) 1 and (1.1) 2 by $\boldsymbol{\varphi}$ and $\boldsymbol{\psi}$, respectively, and integrating formally in $\Omega$, we obtain the following variational formulation for (1.1):

$$
\left\{\begin{array}{l}
\left(\mathbf{y}_{t}, \boldsymbol{\varphi}\right)+a(\mathbf{y}, \boldsymbol{\varphi})+((\mathbf{y} \cdot \nabla) \mathbf{y}, \boldsymbol{\varphi})=(\nabla \times \mathbf{w}, \boldsymbol{\varphi})+\left(\mathbf{u} 1_{\mathcal{O}}, \boldsymbol{\varphi}\right) \\
\left(\mathbf{w}_{t}, \boldsymbol{\psi}\right)+a(\mathbf{w}, \boldsymbol{\psi})-(\nabla(\nabla \cdot \mathbf{w}), \boldsymbol{\psi})+((\mathbf{y} \cdot \nabla) \mathbf{w}, \boldsymbol{\psi})=(\nabla \times \mathbf{y}, \boldsymbol{\psi})+\left(\mathbf{v} 1_{\mathcal{O}}, \boldsymbol{\psi}\right) \\
\mathbf{y}(0)=\mathbf{y}^{0} \in \mathbf{V}, \quad \mathbf{w}(0)=\mathbf{w}^{0} \in \mathbf{H}_{0}^{1}(\Omega)
\end{array}\right.
$$

for all $\{\boldsymbol{\varphi}, \boldsymbol{\psi}\} \in \mathbf{V} \times \mathbf{H}_{0}^{1}(\Omega)$.

\section{Galerkin's approximations}

Let us consider $\left\{\mathbf{e}_{j}\right\}_{j \geq 1}$ and $\left\{\mathbf{f}_{j}\right\}_{j \geq 1}$ bases of $\mathbf{V}$ and $\mathbf{H}_{0}^{1}(\Omega)$, respectively, such that

$$
\left\{\mathbf{e}_{j}\right\}_{j \geq 1} \text { and }\left\{\mathbf{f}_{j}\right\}_{j \geq 1} \text { are linearly independent in } \mathbf{L}^{2}(\mathcal{O}) .
$$

The existence of those bases is guaranteed due to abstract results proved in [23].

We consider the finite-dimensional spaces

$$
E=\operatorname{span}\left[\mathbf{e}_{1}, \ldots, \mathbf{e}_{n}\right] \text { and } F=\operatorname{span}\left[\mathbf{f}_{1}, \ldots, \mathbf{f}_{n}\right] .
$$

Notice that $E \subset \mathbf{V}$ and $F \subset \mathbf{H}_{0}^{1}(\Omega)$.

Let us introduce the Galerkin approximation of the variational formulation (2.2):

$$
\left\{\begin{array}{l}
\left(\mathbf{y}_{t}, \mathbf{e}\right)+a(\mathbf{y}, \mathbf{e})+((\mathbf{y} \cdot \nabla) \mathbf{y}, \mathbf{e})=(\nabla \times \mathbf{w}, \mathbf{e})+\left(\mathbf{u} 1_{\mathcal{O}}, \mathbf{e}\right), \\
\left(\mathbf{w}_{t}, \mathbf{f}\right)+a(\mathbf{w}, \mathbf{f})-(\nabla(\nabla \cdot \mathbf{w}), \mathbf{f})+((\mathbf{y} \cdot \nabla) \mathbf{w}, \mathbf{f})=(\nabla \times \mathbf{y}, \mathbf{f})+\left(\mathbf{v} 1_{\mathcal{O}}, \mathbf{f}\right), \\
\mathbf{y}(0)=\mathbf{y}^{0} \in E, \quad \mathbf{w}(0)=\mathbf{w}^{0} \in F,
\end{array}\right.
$$

for all $\{\mathbf{e}, \mathbf{f}\} \in E \times F$.

System (3.3) has a unique solution $\{\mathbf{y}, \mathbf{w}\} \in C^{0}([0, T] ; E) \times C^{0}([0, T] ; F)$. 
The Galerkin approximation (3.3) is said to be exactly controllable at time $T>0$ if, given $\left\{\mathbf{y}^{0}, \mathbf{w}^{0}\right\},\left\{\mathbf{y}^{T}, \mathbf{w}^{T}\right\} \in E \times F$, there exists a pair of controls $\{\mathbf{u}, \mathbf{v}\} \in$ $\mathbf{L}^{2}(\mathcal{O} \times(0, T))^{2}$ such that the solution $\{\mathbf{y}, \mathbf{w}\}$ of (3.3) satisfies

$$
\{\mathbf{y}(\cdot, T ;\{\mathbf{u}, \mathbf{v}\}), \mathbf{w}(\cdot, T ;\{\mathbf{u}, \mathbf{v}\})\}=\left\{\mathbf{y}^{T}, \mathbf{w}^{T}\right\} .
$$

The cost to achieve (3.4) is given by

$$
C\{\mathbf{u}, \mathbf{v}\}=\frac{1}{2} \int_{\mathcal{O} \times(0, T)}\left(|\mathbf{u}|^{2}+|\mathbf{v}|^{2}\right) d x d t .
$$

The main result of this work is the following:

Theorem 3.1. Let $T>0$ be given. Then the Galerkin approximation (3.3) is exactly controllable in the sense of (3.4). Furthermore, the cost of control given in (3.5) is bounded independently of the nonlinearity.

Proof. Later on, to show and make explicit that the cost of control can be bounded independently of the nonlinearity, we introduce the family of systems

$$
\begin{cases}\mathbf{y}_{t}-\Delta \mathbf{y}+\alpha(\mathbf{y} \cdot \nabla) \mathbf{y}+\nabla p=\nabla \times \mathbf{w}+\mathbf{u} 1_{\mathcal{O}} & \text { in } Q \\ \mathbf{w}_{t}-\Delta \mathbf{w}+\beta(\mathbf{y} \cdot \nabla) \mathbf{w}-\nabla(\nabla \cdot \mathbf{w})=\nabla \times \mathbf{y}+\mathbf{v} 1_{\mathcal{O}} & \text { in } Q, \\ \nabla \cdot \mathbf{y}=0 & \text { in } Q \\ \mathbf{y}=\mathbf{w}=0 & \text { on } \Sigma, \\ \mathbf{y}(0)=\mathbf{y}^{0}, \mathbf{w}(0)=\mathbf{w}^{0} & \text { in } \Omega,\end{cases}
$$

with $\alpha, \beta \in \mathbb{R}$. We will prove some estimates independent of $\alpha$ and $\beta$.

We prove our main result for the system (3.6). First, we introduce its variational formulation. Namely,

$$
\left\{\begin{array}{l}
\left(\mathbf{y}_{t}, \mathbf{e}\right)+a(\mathbf{y}, \mathbf{e})+\alpha((\mathbf{y} \cdot \nabla) \mathbf{y}, \mathbf{e})=(\nabla \times \mathbf{w}, \mathbf{e})+\left(\mathbf{u} 1_{\mathcal{O}}, \mathbf{e}\right) \\
\left(\mathbf{w}_{t}, \mathbf{f}\right)+a(\mathbf{w}, \mathbf{f})+\beta((\mathbf{y} \cdot \nabla) \mathbf{w}, \mathbf{f})-(\nabla(\nabla \cdot \mathbf{w}), \mathbf{f})=(\nabla \times \mathbf{y}, \mathbf{f})+\left(\mathbf{v} 1_{\mathcal{O}}, \mathbf{f}\right) \\
\mathbf{y}(0)=\mathbf{y}^{0} \in E, \quad \mathbf{w}(0)=\mathbf{w}^{0} \in F
\end{array}\right.
$$

for all $\{\mathbf{e}, \mathbf{f}\} \in E \times F$.

We proceed with this proof in several steps.

Step 1 (Linear system). We take a function $\mathbf{h}$ such that

$$
\mathbf{h} \in L^{2}(0, T ; E),
$$

and we consider the linear system

$$
\left\{\begin{array}{l}
\left(\mathbf{y}_{t}, \mathbf{e}\right)+a(\mathbf{y}, \mathbf{e})+\alpha((\mathbf{h} \cdot \nabla) \mathbf{y}, \mathbf{e})=(\nabla \times \mathbf{w}, \mathbf{e})+\left(\mathbf{u} 1_{\mathcal{O}}, \mathbf{e}\right) \\
\left(\mathbf{w}_{t}, \mathbf{f}\right)+a(\mathbf{w}, \mathbf{f})-(\nabla(\nabla \cdot \mathbf{w}), \mathbf{f})+\beta((\mathbf{h} \cdot \nabla) \mathbf{w}, \mathbf{f})=(\nabla \times \mathbf{y}, \mathbf{f})+\left(\mathbf{v} 1_{\mathcal{O}}, \mathbf{f}\right) \\
\mathbf{y}(0)=0, \quad \mathbf{w}(0)=0
\end{array}\right.
$$

for all $\{\mathbf{e}, \mathbf{f}\} \in E \times F$.

System (3.9) has a unique solution $\{\mathbf{y}, \mathbf{w}\} \in C^{0}([0, T] ; E) \times C^{0}([0, T] ; F)$.

Remark 3.2. Due to the linearity of system (3.9), we can assume null initial data. However, all results are valid as well if the initial data is not zero, that is, $\mathbf{y}(0)=$ $\mathbf{y}^{0} \in E$ and $\mathbf{w}(0)=\mathbf{w}^{0} \in F$. 
Let us check that system (3.9) is exactly controllable in any time $T>0$, in the sense of (3.4). For this, it suffices to prove that if $\left\{\mathbf{g}_{1}, \mathbf{g}_{2}\right\} \in E \times F$ satisfies

(3.10) $\left(\{\mathbf{y}(\cdot, T ;\{\mathbf{u}, \mathbf{v}\}), \mathbf{w}(\cdot, T ;\{\mathbf{u}, \mathbf{v}\})\},\left\{\mathbf{g}_{1}, \mathbf{g}_{2}\right\}\right)=0, \forall\{\mathbf{u}, \mathbf{v}\} \in \mathbf{L}^{2}(\mathcal{O} \times(0, T))^{2}$, then $\left\{\mathbf{g}_{1}, \mathbf{g}_{2}\right\} \equiv\{0,0\}$.

Let us consider $\{\boldsymbol{\varphi}, \boldsymbol{\psi}\}$ as being a solution of the adjoint system

$$
\left\{\begin{array}{l}
\left(-\boldsymbol{\varphi}_{t}-\alpha(\mathbf{h} \cdot \nabla) \boldsymbol{\varphi}, \mathbf{e}\right)+a(\boldsymbol{\varphi}, \mathbf{e})=(\nabla \times \boldsymbol{\psi}, \mathbf{e}), \forall \mathbf{e} \in E \\
\left(-\boldsymbol{\psi}_{t}-\beta(\mathbf{h} \cdot \nabla) \boldsymbol{\psi}, \mathbf{f}\right)+a(\boldsymbol{\psi}, \mathbf{f})-(\nabla(\nabla \cdot \boldsymbol{\psi}), \mathbf{f})=(\nabla \times \boldsymbol{\varphi}, \mathbf{f}), \forall \mathbf{f} \in F \\
\boldsymbol{\varphi}(T)=\mathbf{g}_{1} \in E, \quad \boldsymbol{\psi}(T)=\mathbf{g}_{2} \in F
\end{array}\right.
$$

System (3.11) has a unique solution $\{\boldsymbol{\varphi}, \boldsymbol{\psi}\} \in C^{0}([0, T] ; E) \times C^{0}([0, T] ; F)$.

Taking $\{\mathbf{e}, \mathbf{f}\}=\{\mathbf{y}(t), \mathbf{w}(t)\}$ in (3.11) and observing that

$$
((\mathbf{h}(t) \cdot \nabla) \boldsymbol{\varphi}(t), \mathbf{y}(t))=-((\mathbf{h}(t) \cdot \nabla) \mathbf{y}(t), \boldsymbol{\varphi}(t)), \forall t \in[0, T]
$$

(similarly for the term $((\mathbf{h} \cdot \nabla) \boldsymbol{\psi}, \mathbf{w}(t))$ ), we get, after integration by parts in $t$, that

$$
-(\mathbf{y}(T), \boldsymbol{\varphi}(T))+\int_{0}^{T}\left[\left(\mathbf{y}_{t}, \boldsymbol{\varphi}\right)+a(\mathbf{y}, \boldsymbol{\varphi})+\alpha((\mathbf{h} \cdot \nabla) \mathbf{y}, \boldsymbol{\varphi})\right] d t=\int_{0}^{T}(\nabla \times \boldsymbol{\psi}, \mathbf{y}) d t
$$

and

$$
\begin{aligned}
& -(\mathbf{w}(T), \boldsymbol{\psi}(T))+\int_{0}^{T}\left[\left(\mathbf{w}_{t}, \boldsymbol{\psi}\right)+a(\mathbf{w}, \boldsymbol{\psi})+\beta((\mathbf{h} \cdot \nabla) \mathbf{w}, \boldsymbol{\psi})-(\nabla(\nabla \cdot \mathbf{w}), \boldsymbol{\psi})\right] d t \\
& =\int_{0}^{T}(\nabla \times \boldsymbol{\varphi}, \mathbf{w}) d t .
\end{aligned}
$$

Adding the equations (3.12) and (3.13) and taking into account

$$
(\nabla \times \boldsymbol{\psi}, \mathbf{e})=(\boldsymbol{\psi}, \nabla \times \mathbf{e}) \quad \text { and } \quad(\nabla \times \boldsymbol{\varphi}, \mathbf{f})=(\boldsymbol{\varphi}, \nabla \times \mathbf{f}),
$$

we obtain from (3.9) that

$$
\left(\{\mathbf{y}(T), \mathbf{w}(T)\},\left\{\mathbf{g}_{1}, \mathbf{g}_{2}\right\}\right)=\int_{0}^{T}\left(\left\{\mathbf{u} 1_{\mathcal{O}}, \mathbf{v} 1_{\mathcal{O}}\right\},\{\boldsymbol{\varphi}, \boldsymbol{\psi}\}\right) d t .
$$

If (3.10) holds true, then (3.14) implies that

$$
\int_{0}^{T}\left(\left\{\mathbf{u} 1_{\mathcal{O}}, \mathbf{v} 1_{\mathcal{O}}\right\},\{\boldsymbol{\varphi}, \boldsymbol{\psi}\}\right) d t=0, \forall\{\mathbf{u}, \mathbf{v}\} \in \mathbf{L}^{2}(\mathcal{O} \times(0, T))^{2} .
$$

So, one assures

$$
\{\boldsymbol{\varphi}, \boldsymbol{\psi}\}=\{0,0\} \quad \text { in } \quad \mathcal{O} \times(0, T) .
$$

Since $\{\boldsymbol{\varphi}, \boldsymbol{\psi}\}=\sum_{i=1}^{N}\left\{\varphi_{i}(t) \mathbf{e}_{i}, \psi_{i}(t) \mathbf{f}_{i}\right\}$ and thanks to (3.1), we can guarantee by (3.16) that $\left\{\boldsymbol{\varphi}_{i}(t), \boldsymbol{\psi}_{i}(t)\right\}=\{0,0\}$, for $i=1, \ldots, n$. Hence, $\{\boldsymbol{\varphi}, \boldsymbol{\psi}\} \equiv\{0,0\}$ and, therefore, $\left\{\mathbf{g}_{1}, \mathbf{g}_{2}\right\} \equiv\{0,0\}$. Thus, system (3.9) is exactly controllable.

Step 2 (Estimates). Thanks to the results obtained in Step 1, one can define the functional $M: L^{2}(0, T ; E) \rightarrow \mathbb{R}$ by

$$
M(\mathbf{h})=\inf _{\{\mathbf{u}, \mathbf{v}\} \in \mathcal{U}_{a d}} \frac{1}{2} \int_{\mathcal{O} \times(0, T)}\left(|\mathbf{u}|^{2}+|\mathbf{v}|^{2}\right) d x d t,
$$

where $\mathcal{U}_{a d}$ is the set of admissible controls

$$
\mathcal{U}_{a d}=\left\{\{\mathbf{u}, \mathbf{v}\} \in L^{2}(\mathcal{O} \times(0, T))^{2} ;\{\mathbf{y}, \mathbf{w}\} \text { solution of (3.9) satisfying (3.4) }\right\} .
$$


We are interested in proving that

$$
M(\mathbf{h}) \leq \text { constant independent of } \mathbf{h}, \alpha \text { and } \beta .
$$

For this, we use a duality argument.

We consider the continuous linear operator $L: L^{2}(\mathcal{O} \times(0, T))^{2} \rightarrow E \times F$ defined by

$$
L\{\mathbf{u}, \mathbf{v}\}=\{\mathbf{y}(\cdot, T ;\{\mathbf{u}, \mathbf{v}\}), \mathbf{w}(\cdot, T ;\{\mathbf{u}, \mathbf{v}\})\},
$$

and we introduce the functionals

$$
F_{1}\{\mathbf{u}, \mathbf{v}\}=\frac{1}{2} \int_{\mathcal{O} \times(0, T)}\left(|\mathbf{u}|^{2}+|\mathbf{v}|^{2}\right) d x d t
$$

and

$$
F_{2}\left\{\mathbf{g}_{1}, \mathbf{g}_{2}\right\}=\left\{\begin{array}{l}
0, \text { if }\left\{\mathbf{g}_{1}, \mathbf{g}_{2}\right\}=\left\{\mathbf{y}^{T}, \mathbf{w}^{T}\right\} \\
\infty, \text { otherwise. }
\end{array}\right.
$$

In this way, we can rewrite the functional $M$ as follows:

$$
M(\mathbf{h})=\inf _{\{\mathbf{u}, \mathbf{v}\} \in L^{2}(\mathcal{O} \times(0, T))^{2}}\left[F_{1}\{\mathbf{u}, \mathbf{v}\}+F_{2}(L\{\mathbf{u}, \mathbf{v}\})\right] .
$$

From the duality theorem of Fenchel and Rockafellar (see, for instance, [3]), we have

$$
-M(\mathbf{h})=\inf _{\left\{\mathbf{g}_{1}, \mathbf{g}_{2}\right\} \in E \times F}\left[F_{1}^{*}\left(L^{*}\left\{\mathbf{g}_{1}, \mathbf{g}_{2}\right\}\right)+F_{2}^{*}\left(-\left\{\mathbf{g}_{1}, \mathbf{g}_{2}\right\}\right)\right],
$$

where $L^{*}: E \times F \rightarrow L^{2}(\mathcal{O} \times(0, T))^{2}$ is the adjoint of $L$.

Using (3.14), one sees that

$$
L^{*}\left\{\mathbf{g}_{1}, \mathbf{g}_{2}\right\}=\{\boldsymbol{\varphi}, \boldsymbol{\psi}\} \quad \text { in } \mathcal{O} \times(0, T) .
$$

Since

$$
F_{1}^{*}\{\boldsymbol{\varphi}, \boldsymbol{\psi}\}=\frac{1}{2} \int_{\mathcal{O} \times(0, T)}\left(|\boldsymbol{\varphi}|^{2}+|\boldsymbol{\psi}|^{2}\right) d x d t
$$

and

$$
F_{2}^{*}\left(-\left\{\mathbf{g}_{1}, \mathbf{g}_{2}\right\}\right)=-\left(\left\{\mathbf{g}_{1}, \mathbf{g}_{2}\right\},\left\{\mathbf{y}^{T}, \mathbf{w}^{T}\right\}\right),
$$

then

$(3.22)-M(\mathbf{h})=\inf _{\left\{\mathbf{g}_{1}, \mathbf{g}_{2}\right\} \in E \times F}\left[\frac{1}{2} \int_{\mathcal{O} \times(0, T)}\left(|\boldsymbol{\varphi}|^{2}+|\boldsymbol{\psi}|^{2}\right) d x d t-\left(\left\{\mathbf{g}_{1}, \mathbf{g}_{2}\right\},\left\{\mathbf{y}^{T}, \mathbf{w}^{T}\right\}\right)\right]$.

In view of the hypothesis on the basis of $E$ and $F$, we have that $\int_{\mathcal{O}}\left(|\mathbf{e}|^{2}+|\mathbf{f}|^{2}\right) d x$ is a norm on $E \times F$, so that

$$
C\|\{\mathbf{e}, \mathbf{f}\}\|^{2} \geq \int_{\mathcal{O}}\left(|\mathbf{e}|^{2}+|\mathbf{f}|^{2}\right) d x \geq c\|\{\mathbf{e}, \mathbf{f}\}\|^{2}, \forall\{\mathbf{e}, \mathbf{f}\} \in E \times F,
$$

with $c$ and $C$ positive constants that only depend on $E$ and $F$, where

$$
\|\{\mathbf{e}, \mathbf{f}\}\|^{2}=\int_{\Omega}\left(|\mathbf{e}|^{2}+|\mathbf{f}|^{2}\right) d x, \forall\{\mathbf{e}, \mathbf{f}\} \in E \times F .
$$

Thus, (3.22) gives

$$
-M(\mathbf{h}) \geq \inf _{\left\{\mathbf{g}_{1}, \mathbf{g}_{2}\right\} \in E \times F}\left[\frac{c}{2} \int_{Q}\left(|\boldsymbol{\varphi}|^{2}+|\boldsymbol{\psi}|^{2}\right) d x d t-\left(\left\{\mathbf{g}_{1}, \mathbf{g}_{2}\right\},\left\{\mathbf{y}^{T}, \mathbf{w}^{T}\right\}\right)\right] .
$$


Now, we take $\{\mathbf{e}, \mathbf{f}\}=\{\boldsymbol{\varphi}(t), \boldsymbol{\psi}(t)\}$ in (3.11) and integrate from $t$ to $T$. Then the terms containing $\mathbf{h}$ drop out and we obtain

$$
\frac{1}{2}\|\boldsymbol{\varphi}(t)\|^{2}+\int_{t}^{T} a(\boldsymbol{\varphi}, \boldsymbol{\varphi}) d s-\int_{t}^{T}(\nabla \times \boldsymbol{\psi}, \boldsymbol{\varphi}) d s=\frac{1}{2}\left\|\mathbf{g}_{1}\right\|^{2}
$$

and

$$
\frac{1}{2}\|\boldsymbol{\psi}(t)\|^{2}+\int_{t}^{T} a(\boldsymbol{\psi}, \boldsymbol{\psi}) d s-\int_{t}^{T}(\nabla \times \boldsymbol{\psi}, \boldsymbol{\varphi}) d s+\int_{t}^{T} \int_{\Omega}(\nabla \cdot \boldsymbol{\psi})(\nabla \cdot \boldsymbol{\psi}) d x d s=\frac{1}{2}\left\|\mathbf{g}_{2}\right\|^{2} .
$$

Adding the last two equations and integrating in $(0, T)$, we get

$$
\begin{aligned}
& \frac{1}{2} \int_{0}^{T}\left(\|\boldsymbol{\varphi}(t)\|^{2}+\|\boldsymbol{\psi}(t)\|^{2}\right) d t+\int_{0}^{T} t[a(\boldsymbol{\varphi}, \boldsymbol{\varphi})+a(\boldsymbol{\psi}, \boldsymbol{\psi})] d t-2 \int_{0}^{T} t(\nabla \times \boldsymbol{\psi}, \boldsymbol{\varphi}) d t \\
& -\int_{0}^{T} t \int_{\Omega}(\nabla \cdot \boldsymbol{\psi})(\nabla \cdot \boldsymbol{\psi}) d x d t=\frac{T}{2}\left(\left\|\mathbf{g}_{1}\right\|^{2}+\left\|\mathbf{g}_{2}\right\|^{2}\right) .
\end{aligned}
$$

Notice that

$$
a(\boldsymbol{\varphi}(t), \boldsymbol{\varphi}(t))+a(\boldsymbol{\psi}(t), \boldsymbol{\psi}(t)) \leq C\|\{\boldsymbol{\varphi}(t), \boldsymbol{\psi}(t)\}\|^{2}
$$

and

$$
\int_{\Omega}(\nabla \cdot \boldsymbol{\psi}(t))(\nabla \cdot \boldsymbol{\psi}(t)) d x \leq C\|\boldsymbol{\psi}(t)\|^{2}
$$

for some $C>0$ that depends only on $E$ and $F$ because $E$ and $F$ are finite dimensional.

So

$$
\begin{aligned}
& \frac{T}{2}\left(\left\|\mathbf{g}_{1}\right\|^{2}+\left\|\mathbf{g}_{2}\right\|^{2}\right) \leq\left(\frac{1}{2}+C T\right) \int_{0}^{T}\left(\|\boldsymbol{\varphi}(t)\|^{2}+\|\boldsymbol{\psi}(t)\|^{2}\right) d t \\
& +T \int_{0}^{T}\left(a(\boldsymbol{\psi}, \boldsymbol{\psi})+\|\boldsymbol{\varphi}(t)\|^{2}\right) d t+C T \int_{0}^{T}\|\boldsymbol{\psi}(t)\|^{2} d t
\end{aligned}
$$

which implies

$$
\frac{T}{2}\left(\left\|\mathbf{g}_{1}\right\|^{2}+\left\|\mathbf{g}_{2}\right\|^{2}\right) \leq\left(\frac{1}{2}+C T\right) \int_{0}^{T}\left(\|\boldsymbol{\varphi}(t)\|^{2}+\|\boldsymbol{\psi}(t)\|^{2}\right) d t
$$

for a suitable $C>0$ that depends only on $E$ and $F$.

In view of (3.23) we get

$$
-M(\mathbf{h}) \geq \inf _{\left(\mathbf{g}_{1}, \mathbf{g}_{2}\right) \in E \times F}\left[\frac{c T}{(1+2 C T)}\left(\left\|\mathbf{g}_{1}\right\|^{2}+\left\|\mathbf{g}_{2}\right\|^{2}\right)-\left(\left\{\mathbf{g}_{1}, \mathbf{g}_{2}\right\},\left\{\mathbf{y}^{T}, \mathbf{w}^{T}\right\}\right)\right] .
$$

Hence

$$
M(\mathbf{h}) \leq \frac{1+2 C T}{c T}\left(\left\|\mathbf{y}^{T}\right\|^{2}+\left\|\mathbf{w}^{T}\right\|^{2}\right)
$$

which implies (3.18).

Step 3 (Nonlinear system). Let $\mathbf{h} \in L^{2}(0, T ; E)$ be given. We choose for $\{\mathbf{u}, \mathbf{v}\}$ the unique element such that

$$
\frac{1}{2} \int_{\mathcal{O} \times(0, T)}\left(|\mathbf{u}|^{2}+|\mathbf{v}|^{2}\right) d x d t=M(\mathbf{h}) .
$$

We define a continuous mapping $\mathbf{h} \mapsto\{\mathbf{u}, \mathbf{v}\}$ from $L^{2}(0, T ; E)$ in $\mathbf{L}^{2}(\mathcal{O} \times(0, T))^{2}$.

We denote by $\{\mathbf{y}(\mathbf{h}), \mathbf{w}(\mathbf{h})\}$ the solution of (3.9) with the control $\{\mathbf{u}, \mathbf{v}\}=$ $\{\mathbf{u}(\mathbf{h}), \mathbf{v}(\mathbf{h})\}$. 
Taking $\{\mathbf{e}, \mathbf{f}\}=\{\mathbf{y}(t), \mathbf{w}(t)\}$ in (3.9) we obtain

$$
\frac{1}{2} \frac{d}{d t}\|\mathbf{y}(t)\|^{2}+a(\mathbf{y}(t), \mathbf{y}(t))=(\nabla \times \mathbf{w}(t), \mathbf{y}(t))+\left(\mathbf{u} 1_{\mathcal{O}}, \mathbf{y}(t)\right)
$$

and

$\frac{1}{2} \frac{d}{d t}\|\mathbf{w}(t)\|^{2}+a(\mathbf{w}(t), \mathbf{w}(t))+\int_{\Omega}(\nabla \cdot \mathbf{w})(\nabla \cdot \mathbf{w}) d x=(\nabla \times \mathbf{y}(t), \mathbf{w}(t))+\left(\mathbf{v} 1_{\mathcal{O}}, \mathbf{w}(t)\right)$.

In this way, integrating the last two equations in $(0, t)$ and adding them, we have

$$
\begin{aligned}
& \frac{1}{2}\left(\|\mathbf{y}(t)\|^{2}+\|\mathbf{w}(t)\|^{2}\right)+\int_{0}^{t}[a(\mathbf{y}(s), \mathbf{y}(s))+a(\mathbf{w}(s), \mathbf{w}(s))] d s \\
& \leq-\int_{0}^{t} \int_{\Omega}(\nabla \cdot \mathbf{w})(\nabla \cdot \mathbf{w}) d x d s+2 \int_{0}^{t}(\nabla \times \mathbf{w}(s), \mathbf{y}(s)) d s \\
& +\|\mathbf{u}\|_{L^{2}(\mathcal{O} \times(0, T))}\|\mathbf{y}\|_{L^{2}(\mathcal{O} \times(0, t))}+\|\mathbf{v}\|_{L^{2}(\mathcal{O} \times(0, T))}\|\mathbf{w}\|_{L^{2}(\mathcal{O} \times(0, t))}
\end{aligned}
$$

that is,

$$
\begin{aligned}
& \frac{1}{2}\left(\|\mathbf{y}(t)\|^{2}+\|\mathbf{w}(t)\|^{2}\right) \leq C\left(\|\mathbf{u}\|_{\mathbf{L}^{2}(\mathcal{O} \times(0, T))}^{2}+\|\mathbf{v}\|_{\mathbf{L}^{2}(\mathcal{O} \times(0, T))}^{2}\right) \\
& +C \int_{0}^{t}\left(\|\mathbf{y}(s)\|^{2}+\|\mathbf{w}(s)\|^{2}\right) d s .
\end{aligned}
$$

From Gronwall's inequality, we have by (3.31) that

$$
\left(\|\mathbf{y}(t)\|^{2}+\|\mathbf{w}(t)\|^{2}\right) \leq C\left(\|\mathbf{u}\|_{\mathbf{L}^{2}(\mathcal{O} \times(0, T))}^{2}+\|\mathbf{v}\|_{\mathbf{L}^{2}(\mathcal{O} \times(0, T))}^{2}\right) .
$$

In view of (3.18) we obtain that, when $\mathbf{h}$ varies in $L^{2}(0, T ; E)$,

$$
\{\mathbf{y}, \mathbf{w}\} \text { remains a bounded subset } K_{1} \times K_{2} \subset L^{2}(0, T ; E) \times L^{2}(0, T ; F) .
$$

We claim that

$$
\text { the map } \mathbf{h} \mapsto \mathbf{y}(\mathbf{h}) \text { admits a fixed point in } K_{1} \text {. }
$$

Remark 3.3. The application in (3.34) can be seen as being the composition of the applications

and

$$
\mathbf{h} \mapsto\{\mathbf{y}(\mathbf{h}), \mathbf{w}(\mathbf{h})\}
$$

$$
\{\mathbf{y}(\mathbf{h}), \mathbf{w}(\mathbf{h})\} \mapsto \mathbf{y}(\mathbf{h}) .
$$

Assuming for a moment that (3.34) holds, we can conclude the proof of the theorem. In fact, if $\mathbf{h}$ is a fixed point, then, since system (3.9) is exactly controllable in $T>0$, we have the exact controllability of (3.7). Moreover, for any $h$ we have the uniform estimate (3.18), so that the control $\{\mathbf{u}(\mathbf{h}), \mathbf{v}(\mathbf{h})\}$ satisfies the conditions of the theorem.

It remains to prove (3.34).

According to Schauder's fixed point theorem, it suffices to show that the range of $\mathbf{y}(\mathbf{h})$ when $\mathbf{h}$ spans $K_{1}$ is relatively compact in $K_{1}$, which follows from the following estimate:

$$
\mathbf{y}_{t} \text { remains in a bounded set of } L^{2}(0, T ; E) \text { when } h \text { describes } K_{1} \text {. }
$$

To prove (3.35), we observe that, from (3.9), the following holds:

$$
\begin{aligned}
\left|\left(\mathbf{y}_{t}, \mathbf{e}\right)\right| & \leq \alpha\|\mathbf{h}(t)\|\|\nabla \mathbf{y}(t)\|\|\mathbf{e}\|+\|\nabla \mathbf{y}(t)\|\|\nabla \mathbf{e}\|+\|\nabla \mathbf{w}(t)\|\|\mathbf{e}\|+\|\mathbf{u}(t)\|_{\mathbf{L}^{2}(\mathcal{O})}\|\mathbf{e}\|_{\mathbf{L}^{2}(\mathcal{O})} \\
& \leq C\left(\alpha\|\mathbf{h}(t)\|\|\nabla \mathbf{y}(t)\|+\|\nabla \mathbf{y}(t)\|+\|\nabla \mathbf{w}(t)\|+\|\mathbf{u}(t)\|_{\mathbf{L}^{2}(\mathcal{O})}\right)\|\mathbf{e}\|, \quad \forall \mathbf{e} \in E
\end{aligned}
$$


since on the finite-dimensional space $E$ all the norms are equivalent. Therefore

$$
\left\|\mathbf{y}_{t}(t)\right\| \leq C\left(\alpha\|\mathbf{h}(t)\|\|\mathbf{y}(t)\|+\|\mathbf{y}(t)\|+\|\mathbf{w}(t)\|+\|\mathbf{u}(t)\|_{\mathbf{L}^{2}(\mathcal{O})}\right),
$$

which implies (3.35).

Remark 3.4. In the bidimensional case, the nonlinear system which describes the behavior of micropolar fluids (with distributed controls in small sets) reads

$$
\begin{cases}\mathbf{y}_{t}-\Delta \mathbf{y}+(\mathbf{y} \cdot \nabla) \mathbf{y}+\nabla p=\nabla \times w+\mathbf{u} 1_{\mathcal{O}} & \text { in } Q, \\ w_{t}-\Delta w+\mathbf{y} \cdot \nabla w=\nabla \times \mathbf{y}+\mathbf{v} 1_{\mathcal{O}} & \text { in } Q, \\ \nabla \cdot \mathbf{y}=0 & \text { in } Q, \\ \mathbf{y}=0, \quad w=0 & \text { on } \Sigma, \\ \mathbf{y}(0)=\mathbf{y}^{0}, \quad w(0)=w^{0} & \text { in } \Omega .\end{cases}
$$

In this case, the angular velocity $w$ is a scalar variable and the quantities $\nabla \times w$ and $\nabla \times \mathbf{y}$ are defined respectively by

$$
\nabla \times w=\left(\frac{\partial w}{\partial x_{2}},-\frac{\partial w}{\partial x_{1}}\right) \text { and } \nabla \times \mathbf{y}=\frac{\partial \mathbf{y}_{2}}{\partial x_{1}}-\frac{\partial \mathbf{y}_{1}}{\partial x_{2}} .
$$

Using analogous arguments, the Galerkin approximation of (3.37) is also exactly controllable.

\section{REFERENCES}

1. T. Ariman and M. Turk, On steady and pulsatile flow of blood, J. Appl. Mech., 41 (1974), 1-7.

2. J. L. Boldrini, B. Climent-Ezquerra, M. A. Rojas-Medar and M. D. Rojas-Medar, On an Iterative Method for Approximate Solutions of a Generalized Boussinesq Model, to appear in Journal Mathematical Fluid Mechanics.

3. H. Brezis, Analyse fonctionnelle: Theórie et applications, Dunod, Paris, 1999. MR0697382

4. C. Calmelet-Eluhu and D. R. Majumdar, Flow of a micropolar fluid through a circular cylinder subject to longitudinal and torsional oscillations, Math. Comput. Modelling, 27 (8) (1998), 69-78. MR 1625373

5. D. W. Condiff and J. S. Dahler, Fluid mechanics aspects of antisymmetric stress, Phys. Fluids, 11 (1964), 842-854. MR0167060 (29:4333)

6. J.-M. Coron, On the controllability of the 2-D incompressible Navier-Stokes equations with the Navier slip boundary conditions, ESAIM Control Optim. Calc. Var., 1 (1995/96), 35-75. MR 1393067 (97e:93005)

7. J.-M. Coron and A. V. Fursikov, Global exact controllability of the 2D Navier-Stokes equations on a manifold without boundary, Russian J. Math. Phys., 4 (4) (1996), 429-448. MR.1470445 (98k:93057)

8. D. Dupuy, G. P. Panasenko and R. Stavre, Asymptotic methods for micropolar fluids in a tube structure, Math. Models Methods Appl. Sci., 14 (5) (2004), 735-758. MR2057515 (2005b:76035)

9. A. C. Eringen, Theory of micropolar fluids, J. Math. Mech., 16 (1966), 1-18. MR0204005 $(34: 3852)$

10. A. C. Eringen, Simple microfluids, Int. J. Enging. Sci., 2 (1964), 205-217. MR0169468 (29:6716)

11. E. Fernández-Cara and S. Guerrero, Local exact controllability of micropolar fluids, J. Math. Fluid Mech., 9 (2007), 419-453. MR2336077 (2008g:93021)

12. E. Fernández-Cara, S. Guerrero, O. Y. Imanuvilov and J.-P. Puel, Local exact controllability of the Navier-Stokes system, J. Math. Pures Appl., 83 (12) (2004), 1501-1542. MR2103189 (2005g:93013)

13. A. V. Fursikov, Optimal control of distributed systems. Theory and applications, Transl. Math. Monogr., 187, American Mathematical Society, Providence, RI, 2000. MR 1726442 (2000m:49001) 
14. A. V. Fursikov, M. Gunzburger and L. Hou, Optimal boundary control for the evolutionary Navier-Stokes system: the three-dimensional case, SIAM J. Control Optim., 43 (6) (2005), 2191-2232. MR2179484 (2006m:49005)

15. A. V. Fursikov, M. Gunzburger and L. Hou, Optimal Dirichlet control and inhomogeneous boundary value problems for the unsteady Navier-Stokes equations, ESAIM Proc., 4 (1998), 97-116. MR 1663656 (99k:49013)

16. A. V. Fursikov and O. Y. Imanuvilov, Controllability of evolution equations, volume 34 of Lecture Notes Series, Seoul National University Research Institute of Mathematics Global Analysis Center, Seoul, 1996. MR:1406566 (97g:93002)

17. F. Guillén-González, M. A. Rojas-Medar and M. A. Rodríguez-Bellido, Sufficient conditions for regularity and uniqueness of a $3 D$ nematic liquid crystal model, Math. Nachr., 282 (6) (2009), 846-867.

18. O. Y. Imanuvilov, Remarks on exact controllability for the Navier-Stokes equations, ESAIM Control Optim. Calc. Var., 6 (2001), 39-72. MR1804497 (2001k:93013)

19. O. Ladyzhenskaya, The Mathematical Theory of Viscous Incompressible Flow, second edition, Gordon and Breach, New York, 1969. MR0254401 (40:7610)

20. J.-L. Lions, Contrôlabilité exacte, perturbations et stabilisation de systèmes distribués. Tome I. Contrôlabilité Exacte, Rech. Math. Appl. 8, Masson, Paris, 1988. MR963060 (89k:93017)

21. J.-L. Lions and E. Zuazua, Contrôlabilité exacte des approximations de Galerkin des équations de Navier-Stokes, C. R. Acad. Sci. Paris, Ser. I, 234 (1997), 1015-1021. MR.1451243 (98d:93013)

22. J.-L. Lions and E. Zuazua, Exact boundary controllability of Galerkin's approximations of Navier-Stokes equations, Ann. Scuola Norm. Sup. Pisa Cl. Sci., XXVI (4) (1998), 605-621. MR:1648554 (99m:93007)

23. J.-L. Lions and E. Zuazua, On the cost of controlling unstable systems: the case of boundary controls, J. Anal. Math., 73 (1997), 225-249. MR1616414 (98m:93060)

24. G. Lukaszewicz, Micropolar Fluids, Theory and Applications, Modeling and Simulation in Science, Engineering and Technology, Birkhäuser Boston, Inc., Boston, MA, 1999. MR 1711268 (2001a:76013)

25. E. Ortega-Torres, M. A. Rojas-Medar, On the regularity for solutions of the micropolar fluid equations, to appear in Rend. Sem. Mat. Univ. Padova.

26. L. Petrosyan, Some Problems of Fluid Mechanics with Antisymmetric Stress Tensor, Erevan, 1984 (in Russian).

27. A. S. Popel, S. A. Regirer, P. I. Usick, A continuum model of blood flow, Biorheology, 11 (1974), 427-437.

28. M.A. Rojas-Medar, Magneto-micropolar fluid motion: existence and uniqueness of strong solution, Math. Nachr., 188 (1997), 301-319. MR1484679 (99h:76094)

29. R. Stavre, The control of the pressure for a micropolar fluid, Dedicated to Eugen Soós, Z. Angew. Math. Phys., 53 (6) (2002), 912-922. MR1963543 (2004b:76056)

30. R. Temam, Navier-Stokes Equations, Theory and Numerical Analysis ( $2^{\text {nd }}$ Revised Edition), North-Holland, Amsterdam, 1979. MR603444 (82b:35133)

Departamento de Matemática, Universidade Federal da Paraíba, 58051-900, João PesSOA, PB, BRAZIL

E-mail address: fagner@mat.ufpb.br

Departamento de Matemática, Universidade Federal da Paraíba, 58051-900, João PesSOA, PB, BRAZIL

E-mail address: felipewallison@hotmail.com

Departamento de Ciencias Básicas, Universidad del Bío-Bío, Campus Fernando May, Casilla 447, Chillán, Chile

E-mail address: marko@ueubiobio.cl 